Voriconazole more likely than posaconazole increases plasma exposure to sublingual buprenorphine causing a risk of a clinically important interaction

Fihlman, Mari

2016-11

Fihlman, M , Hemmila , T , Hagelberg , N M , Kuusniemi , K, Backman , J T, Laitila , J , Laine , K, Neuvonen, P J , Olkkola , K T \& Saari , T I 2016 , ' Voriconazole more likely than posaconazole increases plasma exposure to sublingual buprenorphine causing a risk of a clinically important interaction ' , European Journal of Clinical Pharmacology , vol. 72 , no. 11 , pp. 1363-1371 . https://doi.org/10.1007/s00228-016-2109-y

http://hdl.handle.net/10138/228315

https://doi.org/10.1007/s00228-016-2109-y

publishedVersion

Downloaded from Helda, University of Helsinki institutional repository.

This is an electronic reprint of the original article.

This reprint may differ from the original in pagination and typographic detail.

Please cite the original version. 


\title{
Voriconazole more likely than posaconazole increases plasma exposure to sublingual buprenorphine causing a risk of a clinically important interaction
}

\author{
Mari Fihlman $^{1,2}$ - Tuija Hemmilä ${ }^{2}$ Nora M. Hagelberg ${ }^{1,2} \cdot$ Kristiina Kuusniemi $^{1,2}$ • \\ Janne T. Backman ${ }^{3} \cdot$ Jouko Laitila $^{3}$ - Kari Laine ${ }^{4,5}$ - Pertti J. Neuvonen ${ }^{3}$ • \\ Klaus T. Olkkola ${ }^{6}$ Teijo I. Saari ${ }^{1,2}$
}

Received: 20 May 2016 / Accepted: 26 July 2016 / Published online: 10 August 2016

(C) Springer-Verlag Berlin Heidelberg 2016

\begin{abstract}
Purpose This study aimed to determine possible effects of voriconazole and posaconazole on the pharmacokinetics and pharmacological effects of sublingual buprenorphine.

Methods We used a randomized, placebo-controlled crossover study design with 12 healthy male volunteers. Subjects were given a dose of $0.4 \mathrm{mg}$ ( $0.6 \mathrm{mg}$ during placebo phase) sublingual buprenorphine after a 5-day oral pretreatment with either (i) placebo, (ii) voriconazole $400 \mathrm{mg}$ twice daily on the first day and $200 \mathrm{mg}$ twice daily thereafter or (iii) posaconazole $400 \mathrm{mg}$ twice daily. Plasma and urine concentrations of buprenorphine and its primary active metabolite norbuprenorphine were monitored over $18 \mathrm{~h}$ and pharmacological effects were measured.
\end{abstract}

Electronic supplementary material The online version of this article (doi:10.1007/s00228-016-2109-y) contains supplementary material, which is available to authorized users.

Teijo I. Saari

teijo.saari@utu.fi

1 Department of Anaesthesiology and Intensive Care, University of Turku, P.O. Box 52, Kiinamyllynkatu 4-8, FI-20521 Turku, Finland

2 Division of Perioperative Services, Intensive Care Medicine and Pain Management, Turku University Hospital, 20521 Turku, Finland

3 Department of Clinical Pharmacology, University of Helsinki and HUSLAB, Helsinki University Central Hospital, 00014 Helsinki, Finland

4 Department of Pharmacology, Drug Development and Therapeutics, University of Turku, 20100 Turku, Finland

5 Medbase Ltd, FI-20100 Turku, Finland

6 Department of Anaesthesiology, Intensive Care and Pain Medicine, University of Helsinki and Helsinki University Hospital, 00014 Helsinki, Finland
Results Compared to placebo, voriconazole increased the mean area under the plasma concentration-time curve $\left(\mathrm{AUC}_{0-\infty}\right)$ of buprenorphine 1.80 -fold $(90 \%$ confidence interval $1.45-2.24 ; P<0.001)$, its peak concentration $\left(\mathrm{C}_{\max }\right) 1.37$ fold $(P<0.013)$ and half-life $\left(t_{1 / 2}\right) 1.37$-fold $(P<0.001)$. Posaconazole increased the $\mathrm{AUC}_{0-\infty}$ of buprenorphine 1.25 -fold $(P<0.001)$. Most of the plasma norbuprenorphine concentrations were below the limit of quantification $(0.05 \mathrm{ng} / \mathrm{ml})$. Voriconazole, unlike posaconazole, increased the urinary excretion of norbuprenorphine 1.58-fold (90\% confidence interval $1.18-2.12 ; P<0.001$ ) but there was no quantifiable parent buprenorphine in urine. Plasma buprenorphine concentrations correlated with the pharmacological effects, but the effects did not differ significantly between the phases.

Conclusions Voriconazole, and to a minor extent posaconazole, increase plasma exposure to sublingual buprenorphine, probably via inhibition of cytochrome P450 $3 \mathrm{~A}$ and/or P-glycoprotein. Care should be exercised in the combined use of buprenorphine with triazole antimycotics, particularly with voriconazole, because their interaction can be of clinical importance.

Keywords Buprenorphine - Azole antifungals .

Voriconazole $\cdot$ Posaconazole $\cdot$ Pharmacokinetics $\cdot$ Drug-drug interaction

\section{Introduction}

Buprenorphine is a semisynthetic partial $\mu$-opioid receptor agonist. The analgesic efficacy of buprenorphine is $20-40$ times higher than that of morphine [1]. Buprenorphine acts also as an antagonist at the $\mathrm{K}$-opioid receptor and as an agonist 
at the $\delta$-opioid receptor and opioid receptor-like receptor. In low doses, it is used in the treatment of moderate acute and chronic pain whereas in high doses, it is used in the management of opioid withdrawal symptoms and opioid dependence $[2,3]$. Buprenorphine can be administered in various formulations and there is a growing interest for its use as an alternative to methadone that could increase access to treatment and be more acceptable to patients $[4,5]$.

After an oral and sublingual administration of buprenorphine, variability in its absorption and disposition increases its susceptibility to drug interactions. Buprenorphine undergoes extensive metabolism, particularly during its firstpass and has an oral bioavailability of $15 \%$ only [6, 7]. Bioavailability following sublingual administration of buprenorphine is higher, but the estimates of absolute bioavailability vary from 15 to $30 \%[8,9]$. Some transporters such as P-glycoprotein can play a role in the pharmacokinetics of buprenorphine and/or its metabolites [10-12]. Peak plasma concentrations of buprenorphine are reached within 1-3 h after sublingual administration [13-15]. Practically no unconjugated parent drug is excreted into urine [14]. The main metabolic pathway, N-dealkylation of buprenorphine, is catalyzed mainly by cytochrome P450 (CYP) 3 A4, and also by CYP3A5 and CYP2C8, yielding an active metabolite norbuprenorphine [16], which is further glucuronidated to norbuprenorphine-3glucuronide [6, 17-20]. In addition to the N-dealkylation, smaller fractions of buprenorphine are converted to other hydroxylated metabolites and to buprenorphine-3-glucuronide. Some in vitro data suggest that CYP2C9 and CYP2C19 are involved in smaller oxidative pathways of buprenorphine [19]. Overall, about 80-90 \% of buprenorphine-derived compounds are excreted by the biliary system and can be subject to enterohepatic circulation [21].

Voriconazole and posaconazole are triazole antifungal agents clinically used in the treatment of disseminated fungal infections. Voriconazole inhibits the activities of CYP3A, CYP2C19, and CYP2C9 enzymes [22], and it has greatly increased plasma concentrations of orally administrated CYP3A4 substrates, e.g., oxycodone [23] and midazolam [24]. Posaconazole is a moderately strong inhibitor of CYP3A4 and CYP2C18, but is not a clinically relevant inhibitor of CYP1A2, CYP2C9, CYP2D6, or CYP2E1 [25, 26]. Concomitant use of posaconazole has increased the plasma concentrations of many drugs that are predominantly metabolized by CYP3A4 [27]. In addition, posaconazole is more potent than voriconazole as inhibitor of P-glycoprotein and breast cancer resistance protein [28].

Some interaction studies have been conducted using highdose buprenorphine and antiretrovirals [29-32]. However, the effect of azole antifungals on the pharmacokinetics of buprenorphine is largely unknown. In particular, there seem to be no previous studies on the possible interaction of voriconazole and posaconazole with sublingual buprenorphine although their concomitant use is likely to occur commonly. We hypothesized that inhibition of CYPmediated metabolism of buprenorphine by voriconazole or posaconazole leads to significant changes in the plasma concentrations of buprenorphine.

\section{Materials and methods}

\section{Study participants}

On the basis of our previous drug-drug interaction studies [33, 34 , we calculated that 10 subjects were needed to detect a $30 \%$ difference in the area under the concentration-time curve $\left(\mathrm{AUC}_{0-\infty}\right)$ of buprenorphine at a power of $80 \%$ and a level of significance of $P<0.05$. To be prepared for dropouts, 12 healthy non-smoking volunteers were recruited in the study. All subjects completed the study. Age and body mass index ranges were 19 to 23 years and 19.8 to $24.8 \mathrm{~kg} / \mathrm{m} 2$, respectively. The inclusion criteria for the volunteers were age 18 to 40 years, body weight within $\pm 15 \%$ of the ideal weight, and blood pressure within normal limits. The criteria for exclusion included a previous history of intolerance to the study drugs; concomitant drug therapy; past or present significant disease or drug allergy, alcoholism, drug abuse, or psychological or emotional problems; blood donation within the 4 weeks prior to the study; lifestyle habits that would compromise the conditions of the study or interpretation of the results. Informed consent was obtained from all individual participants included in the study and the eligible volunteers were ascertained to be healthy by clinical examination, medical history, routine laboratory tests, and an electrocardiogram. It was ensured that urine drug screening was negative. The risk of participants to develop opioid dependency was considered low as evaluated by the Finnish translation of the abuse questions [35].

\section{Study outline and drug administration}

The ethics committee of the Hospital District of Southwest Finland and by the Finnish National Agency for Medicines approved the study protocol, and it was registered in the EudraCT clinical trials register under code number 2010020,953-14. A placebo-controlled, single-blinded, randomized, three-phase crossover study design with 5-day pretreatment periods was used, separated by intervals of 4 weeks. The volunteers ingested orally one of the following treatments in a randomized order: (i) voriconazole (Vfend $200 \mathrm{mg}$ tablet; Pfizer, Sandwich, Great Britain) $400 \mathrm{mg}$ at 8.00 and 20.00 on day $1,200 \mathrm{mg}$ at 8.00 and 20.00 on days 2-4, and $200 \mathrm{mg}$ at 10.00 and 20.00 on day 5 (voriconazole phase), (ii) posaconazole (Noxafil $40 \mathrm{mg} / \mathrm{ml}$ oral suspension; Merck Sharp and Dohme, Hoddesdon, Great Britain) $400 \mathrm{mg}$ at 8.00 
and 20.00 for 4 days and on day 5 , posaconazole was given at 10.00 and 20.00 (posaconazole phase), or (iii) placebo at 8:00 am and 8:00 pm for 4 days, and on day 5 placebo was given at 10.00 and 20.00 (control phase). We assessed the adherence to the premedication schedule using of mobile telephone text messages. On day 5, after fasting overnight, the subjects ingested the premedication with $100 \mathrm{ml}$ of water followed by a single dose of $0.4 \mathrm{mg}(0.6 \mathrm{mg}$ during placebo phase) of sublingual buprenorphine (Temgesic $0.2 \mathrm{mg}$ tablets; RB Pharmaceuticals Limited, Slough, Great Britain) with $20 \mathrm{ml}$ of water $1 \mathrm{~h}$ later. Standardized meals were served 4 and $8 \mathrm{~h}$ after the buprenorphine challenge.

On the test days, a forearm vein was cannulated, and blood samples $(10 \mathrm{ml})$ for pharmacokinetic measurements were collected into ethylenediaminetetraacetic acid-containing tubes immediately before and at the following time points after the administration of buprenorphine: $30 \mathrm{~min}$ and 1, 1.5, 2, 3, 4, 5, $6,8,10,12$, and $18 \mathrm{~h}$. Plasma was separated within $30 \mathrm{~min}$ and stored at $-70{ }^{\circ} \mathrm{C}$ until analysis. Another venous cannula was inserted to the opposite forearm for the possible administration of naloxone (Naloxon B. Braun 0,4 mg/ml, B. Braun Melsungen AG, Melsungen, Germany). Urine was collected up to $18 \mathrm{~h}$ after buprenorphine administration. Urine aliquots were stored at $-70{ }^{\circ} \mathrm{C}$ until analysis. Determination of drug concentrations has been described in detail in Supplement file 1.

\section{Pharmacokinetic measurements}

We observed the peak plasma concentration $\left(C_{\max }\right)$ and time to $C_{\max }\left(t_{\max }\right)$ directly from the data. The area under the plasma concentration-time curve (AUC) values from 0 to $18 \mathrm{~h}$ $\left(\mathrm{AUC}_{0-18}\right)$, as well as from $0 \mathrm{~h}$ to infinity $\left(\mathrm{AUC}_{0-\infty}\right)$, were calculated for buprenorphine applying noncompartmental methods using the WinNonlin pharmacokinetics program (version 4.1; Pharsight, Mountain View, CA). After visual identification of the terminal log-linear part of each concentration-time curve, the elimination rate constant $\left(k_{\mathrm{e}}\right)$ was determined using linear regression analysis. The $t_{1 / 2}$ was calculated using the equation $t_{1 / 2}=\ln 2 / \mathrm{k}_{\mathrm{e}}$. The AUC values were calculated using a combination of the linear and log-linear trapezoidal rules with extrapolation to infinity, when appropriate, by division of the last measured concentration by $k_{\mathrm{e}}$. We also calculated the cumulative amount of free, unconjugated norbuprenorphine excreted into urine from 0 to $18 \mathrm{~h}\left(A_{\mathrm{e}}\right)$.

\section{Measurement of pharmacological effects}

Adverse effects were evaluated using a questionnaire before, and 3 and $6 \mathrm{~h}$ after buprenorphine administration. Other subjective effects of buprenorphine were evaluated using 100$\mathrm{mm}$ visual analog scales for the following items: drowsy/alert, very poor performance/very good performance, no drug effect/very strong drug effect, relaxed/anxious, no nausea/ very strong nausea, calm/restless. The Maddox wing test [36] and Cogan's pupillometer [37] was used to measure the central coordination of extraocular muscles and pupil size, respectively. A digit symbol substitution test was used to estimate central processing of sensory information by recording the number of correct symbols substituted in $3 \mathrm{~min}$ [38]. The analgesic effect was evaluated using the cold pressor test as described earlier [33]. Pharmacological effects were evaluated prior to and at $1,2,3,4,5,6,8,10$, and $12 \mathrm{~h}$ after buprenorphine administration. For each effect variable, area under the response-time (AUEC) curve was determined using the trapezoidal rule.

\section{Statistical analysis}

The data were evaluated for normality of distribution with probit plots and the Shapiro-Wilk's W-test. Log-transformed data were analyzed but nontransformed results are reported. The $\mathrm{AUC}_{0-\infty}$ of buprenorphine was the primary outcome variable in the study, and all other pharmacokinetic and all pharmacodynamic parameters were secondary variables. Geometric mean ratios with $90 \%$ CIs were calculated for the pharmacokinetic variables. Lack of interaction was assumed if the $90 \%$ CI of the geometric mean ratios for pharmacokinetic variables were within the acceptance limit of 0.8 1.25. Pharmacokinetic variables were compared also using repeated-measures analysis of variance with a posteriori testing was performed using the Tukey test. Values for $t_{\max }$ were analyzed using the Wilcoxon signed-ranks test. Differences were regarded as statistically significant when $P<0.05$. The Pearson product moment correlation coefficient was used to investigate the possible relationship between the ratios of the $\mathrm{AUC}_{0-\infty}$ of buprenorphine during the treatment phases (voriconazole or posaconazole) to the $\mathrm{AUC}_{0-\infty}$ of buprenorphine during the control phase, as well as to the $\mathrm{C}_{\text {trough }}$ of voriconazole or posaconazole before the administration of buprenorphine. The associations of plasma buprenorphine concentrations with psychomotor and analgesic effects were also calculated using the Pearson's product moment correlation coefficient. The results are expressed as mean values \pm SD. All data were analyzed using SYSTAT for Windows (version 10.2; Systat Software, Richmond, CA) and R software (version 3.2.0) [39].

\section{Results}

\section{Pharmacokinetics}

The mean plasma concentrations of buprenorphine during the placebo, voriconazole and posaconazole phases are shown in Fig. 1 and the individual amounts of norbuprenorphine 

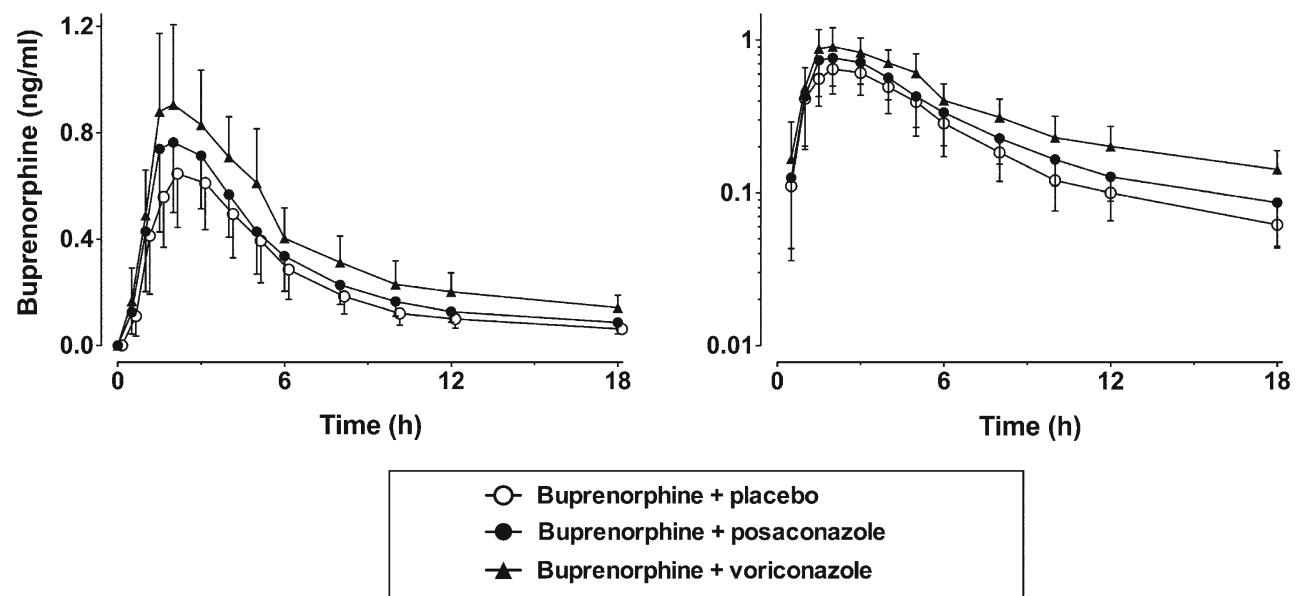

Fig. 1 Mean plasma (SD) concentrations of buprenorphine in 12 healthy volunteers after $0.4 \mathrm{mg}(0.6 \mathrm{mg}$ in placebo phase $)$ sublingual buprenorphine on the fifth day of pretreatment with placebo (open circles), voriconazole $400 \mathrm{mg}$ twice on the first day, thereafter $200 \mathrm{mg}$ twice daily (filled triangles) or posaconazole $400 \mathrm{mg}$ twice daily (filled circles) for 5 days. Right panel shows the same concentrations in semilogarithmic scale. Values are normalized for a sublingual dose of $1.0 \mathrm{mg}$ excreted into urine in Fig. 2. The concentrations of norbuprenorphine were around or below the LLQ in the most of the plasma samples during all three study phases. The effects of voriconazole and posaconazole on the pharmacokinetics of buprenorphine are summarized in Table 1 and Fig. 3.

Effect of voriconazole Compared to the placebo phase, voriconazole increased the mean $A U C_{0-\infty}$ of buprenorphine by 1.80 -fold (90\% confidence interval (CI) 1.45-2.24;

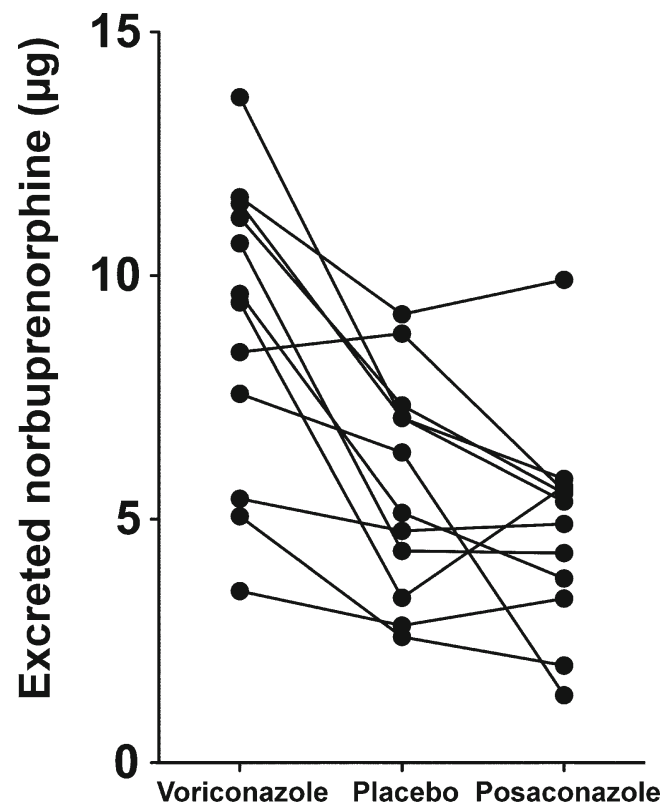

Fig. 2 The individual amounts of urinary norbuprenorphine excreted during $18 \mathrm{~h}$ after $0.4 \mathrm{mg}(0.6 \mathrm{mg}$ in placebo phase) sublingual buprenorphine on the fifth day of pretreatment with placebo, voriconazole ( $400 \mathrm{mg}$ twice on the first day, thereafter $200 \mathrm{mg}$ twice daily) or posaconazole ( $400 \mathrm{mg}$ twice daily) for 5 days in 12 healthy volunteers. Values are normalized for a sublingual dose of $1.0 \mathrm{mg}$
$P<0.001)$ and its $C_{\max }$ by 1.37 -fold (90 \% CI $1.05-1.79$; $P<0.001)$. Voriconazole increased the mean $t_{1 / 2}$ of buprenorphine from 7.9 to $11.0 \mathrm{~h}(P<0.001)$. The mean $C_{\text {trough }}$ of voriconazole on day 5 was $1522 \mathrm{ng} / \mathrm{ml}$ (range $668-4162 \mathrm{ng} / \mathrm{ml})$.

Effect of posaconazole Compared to the placebo phase, posaconazole increased the mean $A U C_{0-\infty}$ of buprenorphine by 1.25 -fold (90\% CI $1.03-1.52 ; P=0.016$ ) and its $C_{\max }$ by 1.20 -fold $(0.97-1.48)$ but the latter effect was not statistically significant $(P=0.206)$. Posaconazole had no effect on the $t_{1 / 2}$ of buprenorphine. The mean $C_{\text {trough }}$ of posaconazole on day 5 was $967 \mathrm{ng} / \mathrm{ml}$ (range $367-1758 \mathrm{ng} / \mathrm{ml}$ ).

Effects on the renal excretion of buprenorphine and norbuprenorphine Voriconazole increased the cumulative amount of norbuprenorphine excreted in urine by 1.6-fold (90 \% CI 1.18-2.12; $P<0.001$ ), when compared to placebo (Table 1). The concentrations of parent buprenorphine in urine were in no study phase reliably quantifiable being much lower than those of norbuprenorphine.

Comparison of voriconazole and posaconazole Voriconazole increased the mean plasma $A U C_{0-18}$ and $A U C_{0-\infty}$ of buprenorphine significantly more than posaconazole $(P<0.001)$. Similarly the cumulative amount of norbuprenorphine excreted to urine was significantly increased during voriconazole, compared to posaconazole $(P<0.001)$.

\section{Pharmacological effects}

Almost every subject experienced some mild or moderate adverse effects (Supplementary Table S1). The most frequent 
Table 1 Pharmacokinetic parameters of buprenorphine after sublingual administration of $0.6 \mathrm{mg}$ (control phase) or $0.4 \mathrm{mg}$ (posaconazole and voriconazole phases) of buprenorphine on the fifth day of pre-treatment with oral posaconazole ( $400 \mathrm{mg}$ twice daily for 5 days), voriconazole $400 \mathrm{mg}$ twice daily on the first day and $200 \mathrm{mg}$ for 4 days) or placebo to 12 healthy volunteers

\begin{tabular}{|c|c|c|c|c|c|c|c|c|c|}
\hline \multirow[t]{2}{*}{ Parameter } & \multirow[t]{2}{*}{ Placebo } & \multirow[t]{2}{*}{ Posaconazole } & \multicolumn{2}{|c|}{ Posaconazole/Placebo } & \multirow[t]{2}{*}{ Voriconazole } & \multicolumn{2}{|c|}{ Voriconazole/placebo } & \multicolumn{2}{|l|}{$\begin{array}{l}\text { Voriconazole } \\
\text { /posaconazole }\end{array}$} \\
\hline & & & $\operatorname{GMR}(90 \% \mathrm{CI})$ & $P$ value & & $\operatorname{GMR}(90 \% \mathrm{CI})$ & $P$ value & GMR $(90 \% \mathrm{CI})$ & $P$ value \\
\hline \multicolumn{10}{|l|}{ Buprenorphine } \\
\hline $\mathrm{C}_{\max }(\mathrm{ng} / \mathrm{ml})$ & $0.70 \pm 0.20$ & $0.84 \pm 0.27$ & $1.20(0.95,1.51)$ & 0.159 & $0.96 \pm 0.27$ & $1.37(1.05,1.79)$ & 0.013 & $1.15(0.93,1.42)$ & 0.333 \\
\hline $\mathrm{t}_{\max }(\mathrm{h})$ & $2.0(1.0-3.0)$ & $2.0(1.5-3.0)$ & - & 0.667 & $2.0(1.5-3.0)$ & - & 0.40 & - & 0.40 \\
\hline $\mathrm{AUC}_{0-18}(\mathrm{ng} . \mathrm{h} / \mathrm{ml})$ & $4.1 \pm 1.2$ & $5.0 \pm 1.3$ & $1.22(1.05,1.41)$ & 0.026 & $6.5 \pm 1.7$ & $1.58(1.28,1.94)$ & $<0.001$ & $1.30(1.10,1.53)$ & 0.003 \\
\hline $\mathrm{AUC}_{0-\infty}(\mathrm{ng} \cdot \mathrm{h} / \mathrm{ml})$ & $4.9 \pm 1.3$ & $6.1 \pm 1.6$ & $1.25(1.20,1.43)$ & 0.008 & $8.9 \pm 2.5$ & $1.80(1.45,2.24)$ & $<0.001$ & $1.44(1.23,1.68)$ & $<0.001$ \\
\hline $\mathrm{Cl} / \mathrm{F}(1 / \mathrm{min})$ & $2.9 \pm 0.9$ & $2.3 \pm 0.7$ & $0.80(0.70,0.91)$ & 0.007 & $1.7 \pm 0.6$ & $0.56(0.48,0.65)$ & $<0.001$ & $0.70(0.60,0.81)$ & $<0.001$ \\
\hline $\mathrm{Vz} / \mathrm{F}(1)$ & $32 \pm 12$ & $27 \pm 11$ & $0.82(0.63,1.10)$ & 0.430 & $25 \pm 11$ & $0.76(0.52,1.12)$ & 0.22 & $0.93(0.64,1.36)$ & 0.892 \\
\hline $\mathrm{t}_{1 / 2}(\mathrm{~h})$ & $7.9 \pm 2.8$ & $8.1 \pm 2.9$ & $1.02(0.77,1.35)$ & 0.991 & $11.0 \pm 3.9$ & $1.37(0.88,2.12)$ & $<0.001$ & $1.34(0.90,2.00)$ & 0.230 \\
\hline \multicolumn{10}{|l|}{ Norbuprenorphine } \\
\hline $\mathrm{A}_{\mathrm{e}}(\mu \mathrm{g})$ & $5.7 \pm 2.2$ & $4.8 \pm 2.2$ & $0.81(0.58,1.14)$ & 0.239 & $9.0 \pm 3.1$ & $1.58(1.18,2.12)$ & $<0.001$ & $1.95(1.45,2.61)$ & $<0.001$ \\
\hline
\end{tabular}

GMR, geometric mean ratio; $\mathrm{CI}$, confidence interval; $\mathrm{C}_{\max }$, peak plasma concentration; $\mathrm{t}_{\max }$, concentration peak time; $\mathrm{AUC}_{0-18}$ and $\mathrm{AUC}_{0-\infty}$, area under curve from 0 to $18 \mathrm{~h}$ and from 0 to infinity, respectively; $\mathrm{t}_{1 / 2}$, elimination half-life; $\mathrm{A}_{\mathrm{e}}$, amount excreted into urine within $18 \mathrm{~h}$. Values are normalized for a sublingual dose of $1.0 \mathrm{mg}$. Data are shown as mean \pm standard deviation (SD) and as the geometric mean ratios with the $90 \%$ confidence interval $(\mathrm{CI})$ in parenthesis - except for $\mathrm{t}_{\max }$, which is given as median and range

adverse effect was sedation, followed by ataxia, dizziness, and nausea. These effects were transient and did not require any treatment.

There was a linear correlation between plasma buprenorphine concentration and pharmacological drug effect $(P<0.001)$. However, there were no statistically significant differences in the pharmacological effects of buprenorphine between the three phases (Supplementary Fig. S1).

\section{Discussion}

This study was designed to investigate the effect of voriconazole and posaconazole on the pharmacokinetics and pharmacodynamics of sublingual buprenorphine in healthy volunteers. The strong CYP3A and CYP2C inhibitor voriconazole markedly increased the $C_{\max }$ and $A U C_{0-\infty}$ of buprenorphine and prolonged its $t_{1 / 2}$. In some subjects, the exposure to buprenorphine was increased more than twofold by voriconazole. On the other hand, the effects of posaconazole on buprenorphine exposure were minor.

We measured also the urinary excretion of norbuprenorphine. As an N-dealkylated metabolite, it is less lipophilic than the parent buprenorphine and can be excreted into urine to some extent also in the unconjugated form. Surprisingly, voriconazole caused a $58 \%$ increase in the amount of norbuprenorphine excreted into urine, although it is reasonable to assume that voriconazole as a strong inhibitor
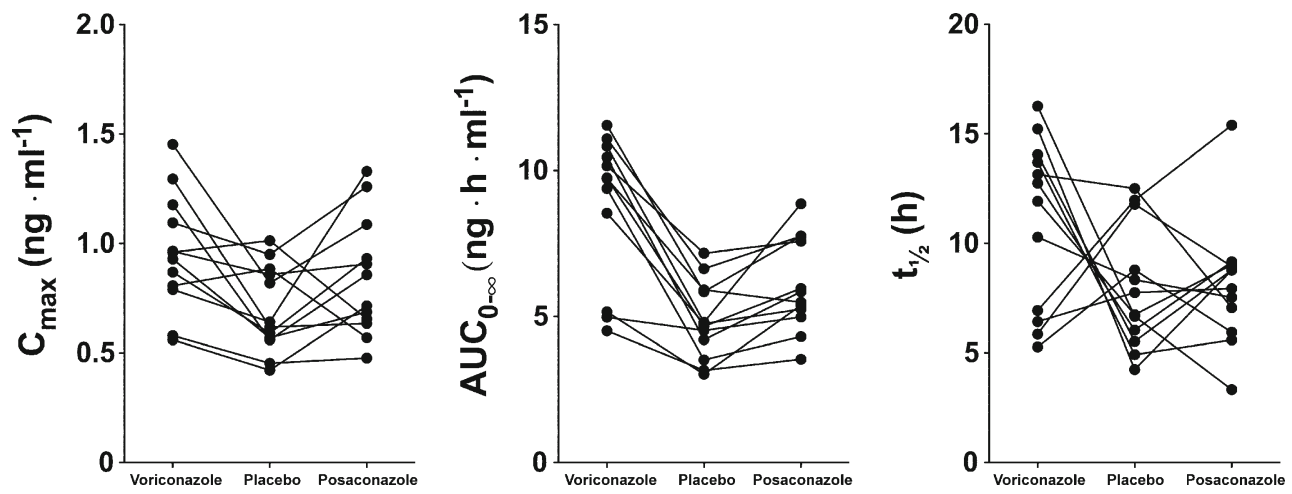

Fig. 3 Individual pharmacokinetic parameters after sublingual buprenorphine. Values for maximum concentration $\left(C_{\max }\right)$, area under plasma concentration-time curve extrapolated to infinity $\left(A U C_{0-\infty}\right)$ and placebo phase) sublingual buprenorphine on the fifth day of pretreatment with placebo, voriconazole (400 $\mathrm{mg}$ twice on the first day, thereafter $200 \mathrm{mg}$ twice daily) or posaconazole ( $400 \mathrm{mg}$ twice daily) for 5 days. elimination half-life $\left(t^{1 / 2}\right)$ in 12 healthy volunteers after $0.4 \mathrm{mg}(0.6 \mathrm{mg}$ in 
of CYP3A4 decreases the (CYP3A4-mediated) Ndealkylation of buprenorphine. On the other hand, the strong and more selective CYP3A4 inhibitor posaconazole actually tended to decrease the excretion of norbuprenorphine into urine, consistent with inhibition of its CYP3A4-mediated formation. Furthermore, norbuprenorphine is a substrate of $\mathrm{P}$ glycoprotein [40]. Different effects of posaconazone (potent inhibitor) and voriconazole (weak inhibitor) on Pglycoprotein may partially explain their different effects on buprenorphine plasma concentrations and on urinary excretion of norbuprenorphine. Unfortunately, we could not determinate the renal clearances, due to low, free, unconjugated concentrations of plasma norbuprenorphine and urine buprenorphine.

It is noteworthy that $400 \mathrm{mg}$ posaconazole twice daily has increased the AUCs of sensitive CYP3A4 substrates several fold; for example, the $A U C$ of oral midazolam was increased about fivefold, consistent with roughly $80 \%$ inhibition of the total CYP3A4-mediated clearance of midazolam [41]. Thus, as posaconazole increased the AUC of buprenorphine by $25 \%$ only, equal to $20 \%$ reduction in oral clearance, it seems that after sublingual buprenorphine dosing, only a small fraction (less than 30-40\%) of buprenorphine dose is metabolized by CYP3A4, and that other CYP-enzymes and UGTs play a larger role. Accordingly, strong inhibition of CYP2C9 and CYP2C19 mediated alternative pathways, in addition to inhibition of CYP3A4-mediated N-demethylation, by voriconazole may partially explain why voriconazole increased the urinary excretion of norbuprenorphine, and increased buprenorphine plasma concentrations more than did posaconazole. In addition, the increased urinary excretion of the metabolite may involve unidentified membrane transporter mechanisms.

Previous studies on drug-drug-interactions between CYPinhibitors and buprenorphine are scarce. The effects of HIV protease inhibitors have been characterized most thoroughly on the pharmacokinetics of high-dose sublingual buprenorphine. Ritonavir increased the $A U C$ of buprenorphine significantly (57\%), while the other protease inhibitors studied had no effect [30-32, 42]. Similarly, the non-nucleoside reverse-transcriptase inhibitors efavirenz and delavirdine increased the AUC of buprenorphine [29]. Atazanavir alone or together with ritonavir increased buprenorphine $A U C_{0-18} 93$ and $67 \%$, respectively, and lead to significant increase in sedative effect [31]. These interactions with buprenorphine are of the same order as those observed in our present study with voriconazole and might necessitate a decrease in the buprenorphine dose during concomitant treatment.

Compared to oral intake, sublingual administration has increased the bioavailability of buprenorphine considerably, from $15 \%$ to up to $30-60 \%[8,9]$. Our present results suggest that strong CYP-inhibitors may further increase the bioavailability of sublingual buprenorphine as both the $C_{\max }$ and $A U C$ of buprenorphine were clearly increased after voriconazole pretreatment. Most probably, voriconazole caused increased exposure by inhibiting both the intestinal and hepatic CYP3A increasing both the $C_{\max }$ and $t_{1 / 2}$ of buprenorphine. In addition, other mechanisms may also be involved. P-glycoprotein is an efflux transporter in the intestinal wall, blood-brain barrier and many other tissues [43]. Several opioids, including morphine [44], fentanyl [45], and alfentanil [46], are substrates of P-glycoprotein, and especially posaconazole is a potent $\mathrm{P}$-glycoprotein inhibitor [28, 47, 48]. Previous studies conducted in transfected cells and mice indicate that P-glycoprotein mediated drug efflux influences brain access and antinociceptive effects of norbuprenorphine [12].

The majority of a buprenorphine dose is excreted as different metabolites into the bile and circulates in enterohepatic system [21]. Voriconazole as a strong inhibitor of many CYP enzymes alters the pharmacokinetics of several drugs metabolized by CYP3A, including oral hypnotics [24, 49, 50] and opioids [33, 51-53]. Voriconazole has decreased the clearance of alfentanil by $83 \%$ [48] and that of fentanyl by $23 \%$ [52], and increased the $A U C_{0-24}$ of R-methadone by $47 \%$ [53]. In the present study, voriconazole increased the exposure to sublingual buprenorphine by 1.8 -fold, i.e., less than has been its effect (tenfold) on oral midazolam [24]. Sublingual buprenorphine partially bypasses the intestinal first-pass metabolism and therefore seems to be less prone than, e.g., oral midazolam or oxycodone to the effects of drugs affecting intestinal and hepatic CYP3A4 during the first-pass metabolism [33, 54, 55].

The half-life of buprenorphine increased significantly after voriconazole, but not after posaconazole pretreatment, which is likely to reflect a decrease in buprenorphine systemic clearance by voriconazole, although an increase in the volume of distribution cannot be excluded without intravenous buprenorphine dosing. The route of administration may affect the apparent half-life of buprenorphine since the estimates appear to be longer after sublingual administration compared to intravenous administration [56, 57]. Furthermore, longer half-lives than observed in the present study have been described after high doses of buprenorphine [8, 15, 56-58]. The terminal elimination phase can continue for a longer time than was the sampling time in the present study, where the limit of buprenorphine quantification after small doses did not allow a longer sampling. Thus, our studies should be interpreted with care in this regard.

In our earlier studies, we have characterized previously unrecognized, drastic increases in the $A U C$ of several substrate drugs, which have a limited therapeutic index, such as midazolam, triazolam, quinidine, tizanidine, and oxycodone, when potent inhibitors of their metabolism have been administered concomitantly [34, 55, 59-62]. Based on these experiences, and for ethical and safety reasons in general, only a 
single clinically relevant, small dose of buprenorphine was employed, because we studied healthy volunteers and we wanted to minimize the risk of adverse events. This may be the reason that the values for the pharmacodynamic variables differed only slightly between phases. Our study was designed mainly to evaluate the pharmacokinetics of buprenorphine, and its power was not sufficient for more precise pharmacodynamic analysis. Nonetheless, we detected a significant linear correlation between plasma buprenorphine concentrations and pharmacological effects, which proves that our methodology was sensitive enough to measure the effects of buprenorphine.

In conclusion, our results show that even a short treatment with clinically used doses of voriconazole increases the exposure to sublingual buprenorphine and this interaction may have a considerable clinical relevance in individual patients. When the interindividual variation in the pharmacokinetics of buprenorphine is taken into account, some individuals given voriconazole may have a more than $100 \%$ increase in the exposure to buprenorphine. Thus, at least the interaction between voriconazole and sublingual buprenorphine can be of clinical significance. It has been previously shown that the strong CYP3A-inhibitor ketoconazole had no effect on the pharmacokinetics of transdermal buprenorphine [63]. Our results warrant careful patient monitoring when sublingual buprenorphine is used with triazole antifungals, especially voriconazole. Posaconazole is less likely to cause a clinically significant interaction with sublingual buprenorphine.

Acknowledgments We thank Mrs. Elina Kahra (medical laboratory technologist, Clinical Pharmacology, TYKSLAB, Hospital District of Southwest Finland, Turku, Finland) for her skillful technical assistance. This study was supported financially by Turku University Hospital research fund (EVO 13821), Turku, Finland.

\section{Compliance with ethical standards}

Authors' contributions Mari Fihlman took care of the clinical phase of the study and data collection, participated in data analysis and statistical analysis and wrote the manuscript. Klaus Olkkola and Kari Laine designed the study, wrote the protocol, supervised and coordinated the clinical implementation of the study, and participated in data analysis. Tuija Hemmilä and Kristiina Kuusniemi participated the clinical phase and data collection. Janne T. Backman, Jouko Laitila and Pertti J Neuvonen performed the analytical assays. Teijo Saari designed the study, analyzed the data, performed statistical analysis, and wrote the manuscript. All authors materially participated in the research and/or manuscript preparation. All authors have contributed to and approved the final manuscript.

Conflict of interest The authors declare that they have no conflict of interest.

\section{References}

1. Cowan A (1995) Update on the general pharmacology of buprenorphine. In: Cowan A, Lewis J (eds) Buprenorphine combat. Drug Abus. With a unique opioid. Wiley-Liss, New York, pp. 189211

2. Mattick RP, Ali R, White JM, et al. (2003) Buprenorphine versus methadone maintenance therapy: a randomized double-blind trial with 405 opioid-dependent patients. Addiction 98:441-452

3. Petitjean S, Stohler R, Déglon JJ, et al. (2001) Double-blind randomized trial of buprenorphine and methadone in opiate dependence. Drug Alcohol Depend 62:97-104

4. Yarborough BJH, Stumbo SP, McCarty D, et al. (2016) Methadone, buprenorphine and preferences for opioid agonist treatment: a qualitative analysis. Drug Alcohol Depend 160:112-118. doi:10.1016/j. drugalcdep.2015.12.031

5. Gryczynski J, Jaffe JH, Schwartz RP, et al. (2013) Patient perspectives on choosing buprenorphine over methadone in an urban, equal-access system. Am J Addict 22:285-291

6. Cone EJ, Gorodetzky CW, Yousefnejad D, et al. (1984) The metabolism and excretion of buprenorphine in humans. Drug Metab Dispos 12:577-581

7. Elkader A, Sproule B (2005) Buprenorphine: clinical pharmacokinetics in the treatment of opioid dependence. Clin Pharmacokinet 44:661-680

8. Mendelson J, Upton RA, Everhart ET, et al. (1997) Bioavailability of sublingual buprenorphine. J Clin Pharmacol 37:31-37

9. Nath RP, Upton RA, Everhart ET, et al. (1999) Buprenorphine pharmacokinetics: relative bioavailability of sublingual tablet and liquid formulations. J Clin Pharmacol 39:619-623

10. Alhaddad H, Cisternino S, Declèves X, et al. (2012) Respiratory toxicity of buprenorphine results from the blockage of $\mathrm{P}$ glycoprotein-mediated efflux of norbuprenorphine at the bloodbrain barrier in mice. Crit Care Med 40:3215-3223

11. Hassan HE, Myers AL, Coop A, Eddington ND (2009) Differential involvement of P-glycoprotein (ABCB1) in permeability, tissue distribution, and antinociceptive activity of methadone, buprenorphine, and Diprenorphine: in vitro and in vivo evaluation. J Pharm Sci 98:4928-4940

12. Brown SM, Campbell SD, Crafford A, et al. (2012) P-glycoprotein is a major determinant of norbuprenorphine brain exposure and antinociception. J Pharmacol Exp Ther 343:53-61. doi:10.1124 /jpet.112.193433

13. McAleer SD, Mills RJ, Polack T, et al. (2003) Pharmacokinetics of high-dose buprenorphine following single administration of sublingual tablet formulations in opioid nave healthy male volunteers under a naltrexone block. Drug Alcohol Depend 72:75-83

14. Ciraulo DA, Hitzemann RJ, Somoza E, et al. (2006) Pharmacokinetics and pharmacodynamics of multiple sublingual buprenorphine tablets in dose-escalation trials. J Clin Pharmacol 46:179-192

15. Bullingham RE, McQuay HJ, Dwyer D, et al. (1981) Sublingual buprenorphine used postoperatively: clinical observations and preliminary pharmacokinetic analysis. Br J Clin Pharmacol 12:117122

16. Brown SM, Holtzman M, Kim T, Kharasch ED (2011) Buprenorphine metabolites, buprenorphine-3-glucuronide and norbuprenorphine-3-glucuronide, are biologically active. Anesthesiol 115:1251-1260

17. Iribarne C, Picart D, Dréano Y, et al. (1997) Involvement of cytochrome P450 3 A4 in N-dealkylation of buprenorphine in human liver microsomes. Life Sci 60:1953-1964

18. Kobayashi K, Yamamoto T, Chiba K, et al. (1998) Human buprenorphine $\mathrm{N}$-dealkylation is catalyzed by cytochrome $\mathrm{P} 450$ 3 A4. Drug Metab Dispos 26:818-821 
19. Moody DE, Slawson MH, Strain EC, et al (2002) A liquid chromatographic-electrospray ionization-tandem mass spectrometric method for determination of buprenorphine, its metabolite, norbuprenorphine, and a coformulant, naloxone, that is suitable for in vivo and in vitro metabolism studies. Anal Biochem 306: 31-39

20. Picard N, Cresteil T, Djebli N, Marquet P (2005) In vitro metabolism study of buprenorphine: evidence for new metabolic pathways. Drug Metab Dispos 33:689-695. doi:10.1124/dmd.105.003681

21. Brewster D, Humphrey MJ, McLeavy MA (1981) Biliary excretion, metabolism and enterohepatic circulation of buprenorphine. Xenobiotica 11:189-196

22. Niwa T, Shiraga T, Takagi A (2005) Effect of antifungal drugs on cytochrome P450 (CYP) 2C9, CYP2C19, and CYP3A4 activities in human liver microsomes. Biol Pharm Bull 28:1805-1808

23. Hagelberg NM, Nieminen TH, Saari TI, et al. (2009) Voriconazole drastically increases exposure to oral oxycodone. Eur J Clin Pharmacol 65:263-271

24. Saari TI, Laine K, Leino K, et al. (2006) Effect of voriconazole on the pharmacokinetics and pharmacodynamics of intravenous and oral midazolam. Clin Pharmacol Ther 79:362-370

25. Wexler D, Courtney R, Richards W, et al. (2004) Effect of posaconazole on cytochrome P450 enzymes: a randomized, openlabel, two-way crossover study. Eur J Pharm Sci 21:645-653

26. Moody DE, Liu F, Fang WB (2015) Azole antifungal inhibition of buprenorphine, methadone and oxycodone in vitro metabolism. $\mathrm{J}$ Anal Toxicol 39:374-386

27. Li Y, Theuretzbacher U, Clancy CJ, et al. (2010) Pharmacokinetic/ pharmacodynamic profile of posaconazole. Clin Pharmacokinet 49: 379-396

28. Lempers VJC, van den Heuvel JJMW, Russel FGM, et al. (2016) Inhibitory potential of antifungal drugs on ATP-binding cassette transporters P-glycoprotein, MRP1 to MRP5, BCRP, and BSEP. Antimicrob Agents Chemother 60:3372-3379

29. McCance-Katz EF, Moody DE, Morse GD, et al. (2006) Interactions between buprenorphine and antiretrovirals. I. The nonnucleoside reverse-transcriptase inhibitors efavirenz and delavirdine. Clin Infect Dis 43(Suppl 4):S224-S234

30. McCance-Katz EF, Moody DE, Smith PF, et al. (2006) Interactions between buprenorphine and antiretrovirals. II. The protease inhibitors nelfinavir, lopinavir/ritonavir, and ritonavir. Clin Infect Dis 43(Suppl 4):S235-S246

31. McCance-Katz EF, Moody DE, Morse GD, et al. (2007) Interaction between buprenorphine and atazanavir or atazanavir/ritonavir. Drug Alcohol Depend 91:269-278

32. Hulskotte EGJ, Bruce RD, Feng H-P, et al. (2015) Pharmacokinetic interaction between HCV protease inhibitor boceprevir and methadone or buprenorphine in subjects on stable maintenance therapy. Eur J Clin Pharmacol 71:303-311

33. Nieminen TH, Hagelberg NM, Saari TI, et al. (2009) Rifampin greatly reduces the plasma concentrations of intravenous and oral oxycodone. Anesthesiol 110:1371-1378

34. Saari TI, Grönlund J, Hagelberg NM, et al. (2010) Effects of itraconazole on the pharmacokinetics and pharmacodynamics of intravenously and orally administered oxycodone. Eur J Clin Pharmacol 66:387-397

35. Michna E, Ross EL, Hynes WL, et al. (2004) Predicting aberrant drug behavior in patients treated for chronic pain: importance of abuse history. J Pain Symptom Manag 28:250-258

36. Hannington-Kiff JG (1970) Measurement of recovery from outpatient general anaesthesia with a simple ocular test. Br Med J 3:132135

37. Cogan D (1941) A simplified entoptic pupillometer. Am J Ophthalmol 24:1431-1433

38. Stone BM (1984) Pencil and paper tests-sensitivity to psychotropic drugs. Br J Clin Pharmacol 18(Suppl 1):15S-20S
39. R Foundation for Statistical Computing (2015) R: a language and environment for statistical computing

40. Tournier N, Chevillard L, Megarbane B, et al. (2010) Interaction of drugs of abuse and maintenance treatments with human $\mathrm{P}$ glycoprotein $(\mathrm{ABCB} 1)$ and breast cancer resistance protein (ABCG2). Int J Neuropsychopharmacol 13:905-915

41. Krishna G, Moton A, Ma L, et al. (2009) Effects of oral posaconazole on the pharmacokinetic properties of oral and intravenous midazolam: a phase I, randomized, open-label, crossover study in healthy volunteers. Clin Ther 31:286-298

42. Bruce RD, Altice FL, Moody DE, et al. (2010) Pharmacokinetic interactions between buprenorphine/naloxone and once-daily lopinavir/ritonavir. J Acquir Immune Defic Syndr 54:511-514

43. DuBuske LM (2005) The role of P-glycoprotein and organic aniontransporting polypeptides in drug interactions. Drug Saf 28:789801

44. Kharasch ED, Hoffer C, Whittington D, Sheffels P (2003) Role of P-glycoprotein in the intestinal absorption and clinical effects of morphine. Clin Pharmacol Ther 74:543-554

45. Kharasch ED, Hoffer C, Altuntas TG, Whittington D (2004) Quinidine as a probe for the role of p-glycoprotein in the intestinal absorption and clinical effects of fentanyl. J Clin Pharmacol 44: 224-233

46. Kalvass JC, Olson ER, Pollack GM (2007) Pharmacokinetics and pharmacodynamics of alfentanil in P-glycoprotein-competent and P-glycoprotein-deficient mice: P-glycoprotein efflux alters alfentanil brain disposition and antinociception. Drug Metab Dispos 35:455-459

47. Sansone-Parsons A, Krishna G, Simon J, et al. (2007) Effects of age, gender, and race/ethnicity on the pharmacokinetics of posaconazole in healthy volunteers. Antimicrob Agents Chemother 51:495-502

48. Ashbee HR, Gilleece MH (2012) Has the era of individualised medicine arrived for antifungals? A review of antifungal pharmacogenomics. Bone Marrow Transplant 47:881-894

49. Saari TI, Laine K, Leino K, et al. (2006) Effect of voriconazole on the pharmacokinetics and pharmacodynamics of zolpidem in healthy subjects. Br J Clin Pharmacol 63:116-120

50. Saari TI, Laine K, Bertilsson L, et al. (2007) Voriconazole and fluconazole increase the exposure to oral diazepam. Eur J Clin Pharmacol 63:941-949

51. Saari TI, Laine K, Leino K, et al. (2006) Voriconazole, but not terbinafine, markedly reduces alfentanil clearance and prolongs its half-life. Clin Pharmacol Ther 80:502-508

52. Saari TI, Laine K, Neuvonen M, et al. (2008) Effect of voriconazole and fluconazole on the pharmacokinetics of intravenous fentanyl. Eur J Clin Pharmacol 64:25-30

53. Liu P, Foster G, Labadie R, et al. (2007) Pharmacokinetic interaction between voriconazole and methadone at steady state in patients on methadone therapy. Antimicrob Agents Chemother 51:110-118

54. Olkkola KT, Backman JT, Neuvonen PJ (1994) Midazolam should be avoided in patients receiving the systemic antimycotics ketoconazole or itraconazole. Clin Pharmacol Ther 55:481-485

55. Olkkola KT, Ahonen J, Neuvonen PJ (1996) The effects of the systemic antimycotics, itraconazole and fluconazole, on the pharmacokinetics and pharmacodynamics of intravenous and oral midazolam. Anesth Analg 82:511-516

56. Bullingham RE, McQuay HJ, Moore A, Bennett MR (1980) Buprenorphine kinetics. Clin Pharmacol Ther 28:667-672

57. Kuhlman JJ, Lalani S, Magluilo J, et al. (1996) Human pharmacokinetics of intravenous, sublingual, and buccal buprenorphine. J Anal Toxicol 20:369-378

58. Kuhlman JJ, Levine B, Johnson RE, et al. (1998) Relationship of plasma buprenorphine and norbuprenorphine to withdrawal symptoms during dose induction, maintenance and withdrawal from sublingual buprenorphine. Addiction 93:549-559 
59. Kaukonen KM, Olkkola KT, Neuvonen PJ (1997) Itraconazole increases plasma concentrations of quinidine. Clin Pharmacol Ther 62:510-517

60. Varhe A, Olkkola KT, Neuvonen PJ (1994) Oral triazolam is potentially hazardous to patients receiving systemic antimycotics ketoconazole or itraconazole. Clin Pharmacol Ther 56:601-607

61. Backman JT, Kivistö KT, Olkkola KT, Neuvonen PJ (1998) The area under the plasma concentration-time curve for oral midazolam is 400 -fold larger during treatment with itraconazole than with rifampicin. Eur J Clin Pharmacol 54:53-58
62. Granfors MT, Backman JT, Neuvonen M, et al. (2004) Fluvoxamine drastically increases concentrations and effects of tizanidine: a potentially hazardous interaction. Clin Pharmacol Ther 75:331-341

63. Kapil RP, Cipriano A, Michels GH, et al. (2012) Effect of ketoconazole on the pharmacokinetic profile of buprenorphine following administration of a once-weekly buprenorphine transdermal system. Clin Drug Investig 32:583-592 